\title{
DYNAMIC SIMULATION CONTROL IN A CRYOGENIC DISTILLATION COLUMN
}

\author{
${ }^{1}$ Viktória KÁLLAI* ${ }^{2}$ Gábor L. SZEPESI, ${ }^{3}$ Péter MIZSEY \\ ${ }^{1,2}$ Institute of Energy Engineering and Chemical Machinery \\ Faculty of Mechanical Engineering and Informatics, University of Miskolc \\ Miskolc-Egyetemváros, H-3515 Miskolc, Hungary \\ e-mail: 1vegykalv@uni-miskolc.hu, ${ }^{2}$ szepesi@uni-miskolc.hu \\ ${ }^{3}$ Institute of Chemistry, Faculty of Material Science and Engineering \\ University of Miskolc, Miskolc-Egyetemváros, H-3515 Miskolc, Hungary \\ e-mail: kemizsey@uni-miskolc.hu
}

Received 30 December 2019; accepted 16 March 2020

\begin{abstract}
Chemical industry has a high demand for ethylene quantity, especially with high quality. This paper discusses dynamic simulation models of an ethaneethylene high-pressure cryogenic rectification column with Unisim Design process simulator software. Distillation is one of the most essential technologies in chemical industry, it is important that the operation of the procedure can be modeled not only in steady-state mode but also in a dynamic way. The goal during this study is to make simulations with system-controlling and to investigate the effect the disturbance on the behavior of the columns.
\end{abstract}

Keywords: Dynamic simulation, Rectification column, Unisim design code, Controlling

\section{Introduction}

Distillation is a separation technology, which is based on the different boiling points (or volatilities) of the components mixture. This process is really energy- and costintensive, and frequently used in chemical industry [1]. The equipment of this technology is the column; furthermore, a reboiler and a condenser [2] also belong to this system. The tower has one feed stream and two product streams. The more volatile component is enriched in the vapor fraction and leaves the column as the overhead product, while the less volatile component is enriched in the liquid phase and goes down and down and leaves the tower like the bottom product [3]. During the distillation

${ }^{*}$ Corresponding Author 
procedure, the goal is to separate from each other the components and to reach the products in high purity.

The distillation is the most studied unit operation in terms of control [4]. For controlling the system Proportional-Integral-Derivative (PID) controllers were used [5]. This is the oldest and widely used feedback control type for different industrial processes. A PID controller computes a so-called error value $(E)$ when there is a difference between the measured Process Value $(P V)$ and required SetPoint values $(S P)$ of the processes. The exercise of the controller is to bring the error equal or close to zero by the control variable value, the OutPut value $(O P)$. The PID controller consists of three terms: the Proportional term $(P)$, (it depends on the present error), the Integral term $(I)$, (it depends on the sum of past errors), the Derivative term $(D)$, (this is the prediction of future errors) [6]-[9].

The equation of the transfer function of the PID controller is (all variable is based on time parameter):

$$
O P(t)=K\left(E(t)+\frac{1}{T_{i}} \int_{0}^{t} E(\tau) d \tau+T_{d} \frac{d E(t)}{d t}\right)
$$

where $K$ is the proportional gain; $T_{i}$ is the integral time; and $T_{d}$ is the derivative time.

The using of the P-term results in faster controlling process, but difference can be observed between the setpoint and the process variable values. However, using the controlling process with the $I$ - and $D$-terms the controlling process is slower, and there is no difference between the setpoint and process variable values [6].

It is also possible to use not only together all of the $P$-, $I$ - and $D$ - terms, but also separate the $P, P I, P D$ or $P I D$ types of controllers [10].

During a controlling process of a one-feed and two-product distillation column 5 control valves can be positioned. The manipulated parameters can be the levels in top and bottom, pressure and composition in top and bottom. There are two general types of controlling structures: level controlling (top and bottom levels, pressure) and composition controlling (top and bottom compositions). The first type should be used in the interest of stable operation and because it is independent from the composition control loop, it is designed first. The measured parameters were chosen according to the practices and literature of distillation controlling [11], [12].

In this study two types of controlling structure were used, however the temperature controlling was used instead of the pressure controlling, because of the unequivocal connection between temperature and pressure. From the temperature profile the position of the temperature measurement was given. The first type of control structure consisted the one temperature and two level controllers. The second type of the control structure besides these controllers - consisted the top product controller. During this study only composition of the top product was used, because according to the separation the essential product is the overhead product, it has many possible usages.

\section{Parameters of the investigated system}

During this study, an ethane-ethylene separation column was investigated. In the chemical industry, ethylene is polymerized and used as one of the most important raw 
material of plastic manufacturing. Ethane is usually recycled to the beginning of the procedure. It can be used as a refrigerant in the system [13].

The mixture - ethane and ethylene - is a zeotropic one; therefore the ethane and the ethylene can be separated from each other's. Fig. 1 shows the vapor-liquid equilibrium diagram of the mixture, when the pressure is 8 bar.

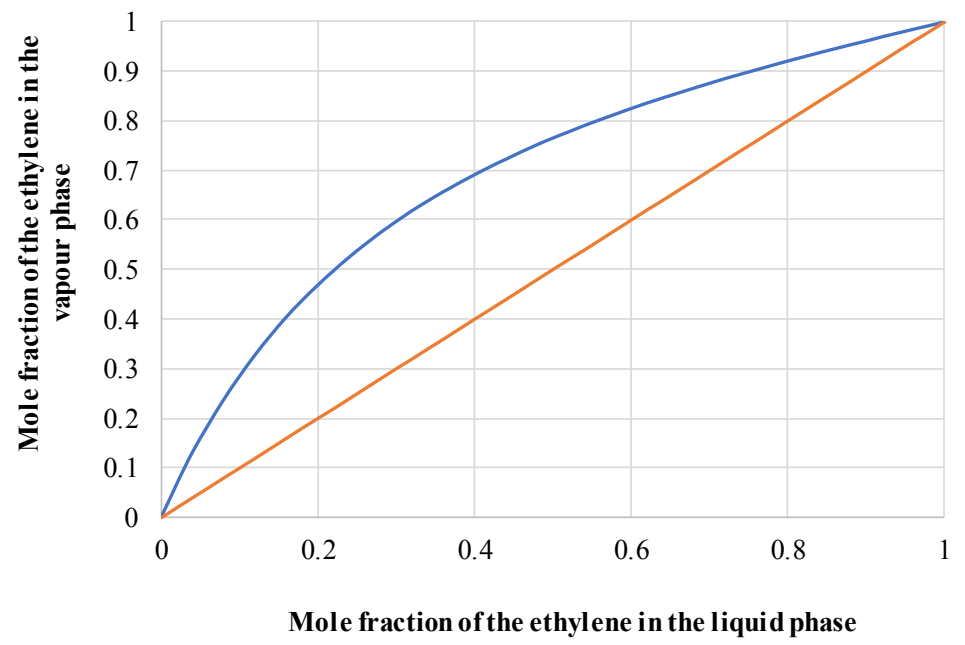

Fig. 1. Vapor-liquid equilibrium diagram of the components

In Table I the parameters of the investigated rectification column are summarized.

Table I

Parameters of the rectification column

\begin{tabular}{|l|l|}
\hline Number of stages & 100 \\
Feed stage & $75^{\text {th }}$ \\
Reflux ratio & 2.2 \\
Heat flow of the condense $[\mathrm{kW}]$ & 15320 \\
Heat flow of the reboiler $[\mathrm{kW}]$ & 9132 \\
\hline
\end{tabular}

Unisim Design [14] process simulator software with Soave-Redlich-Kwong (SRK) fluid package [15], [16] was used for making simulations. Firstly, the simulation was prepared in steady-state mode. Table II contains the technical data of the feed stream and the results of the simulation (parameters of the distillate and bottom product).

From the steady-state model, the temperature profile (Fig. 2) of the column was also illustrated in case of different ethylene contents in the overhead product $\left(x_{D}=0.9-0.9995\right)$. In the stripping zone, all types of curves have the same shape; however, in the rectifying zone the profile of the curves are different. 
Table II

Streams of the parameters of the column

\begin{tabular}{|l|l|l|l|}
\hline & Feed & Distillate & Bottom product \\
\hline Temperature $\left[{ }^{\circ} \mathrm{C}\right]$ & -55 & -61 & -40 \\
Pressure $[\mathrm{bar}]$ & 7.95 & 7.48 & 7.86 \\
Mass flow $[\mathrm{kg} / \mathrm{h}]$ & 53750 & 44190 & 9560 \\
Composition $[\mathrm{n} / \mathrm{n}]$ & & & \\
Ethylene & 0.833 & 0.9995 & 0.0073 \\
Ethane & 0.167 & 0.0005 & 0.9927 \\
\hline
\end{tabular}

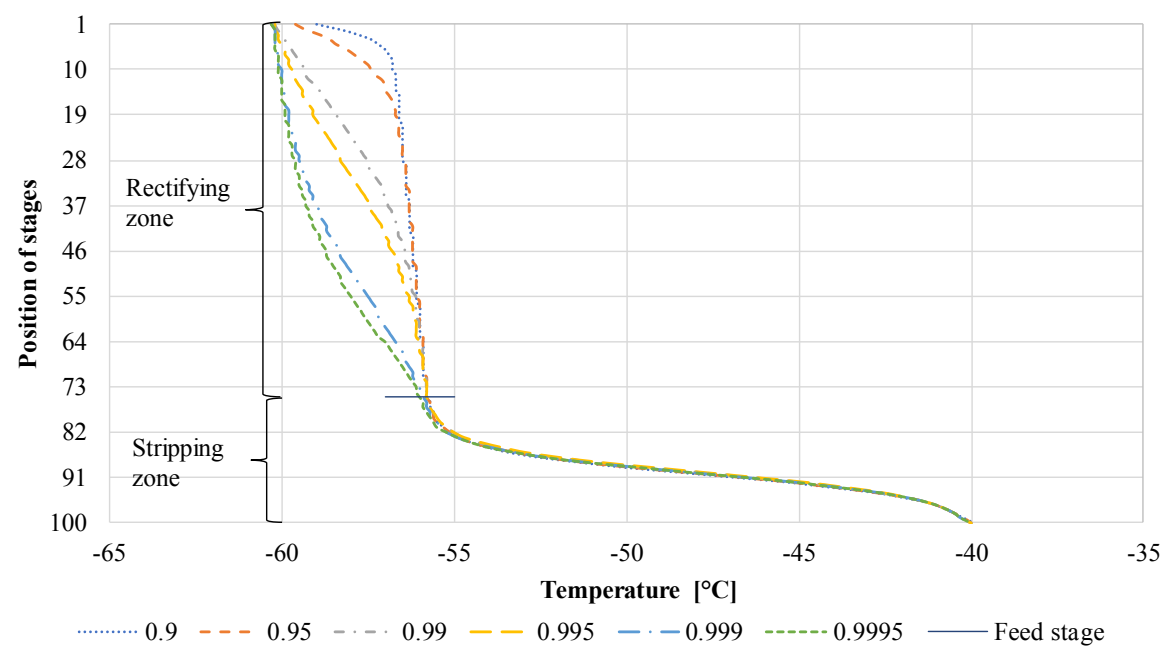

Fig. 2. Temperature profiles

This system had six degrees of freedom. These are summarized in Table III.

Table III

Degrees of freedom

\begin{tabular}{|l|l|}
\hline Degree of freedom & Value \\
\hline Temperature of the feed stream & $-55^{\circ} \mathrm{C}$ \\
Pressure of the feed stream & $7.95 \mathrm{bar}$ \\
Mass flow of the feed stream & $53750 \mathrm{~kg} / \mathrm{h}$ \\
Ethylene mole fraction of the feed stream & 0.833 \\
Reflux ratio & 2.2 \\
Ethylene content in the distillate stream & 0.9995 \\
\hline
\end{tabular}

From the curves it can be determined that the two smallest values of the ethylene purification (0.9 and 0.95) are almost straight approximately throughout $30^{\circ} \mathrm{C}$ interval, therefore these have a sharp increase. The next two cases have also a relatively rapid 
temperature growing. From the viewpoint of the operation of the separation the curves with two highest values have the most appropriate shape. According to the curve shape of the original purification of the overhead product, in this study the temperature controller was situated on the $70^{\text {th }}$ tray. It should not be far from the location of the output variable (in this case the heat flow of the reboiler was that) [17].

\section{Dynamic simulation models and influences of the used disturbances}

\subsection{First investigated dynamic simulation model}

The dynamic models were also made with the Unisim Design process simulator software. The goal of this study was the investigating of the behavior of the rectification column. For that two types of disturbances were investigated: the mass flow of the feed stream was decreased and increased. Firstly, three PI controllers were used in the model (Fig. 3):

- two level controllers (LIC-100 and LIC-101): these controlled the liquid level in the condenser or reboiler to get the appropriate mass flow of the distillate or the bottom product;

- a temperature controller (TIC-100): the manipulated parameter was the heat flow of the reboiler, and the goal was to hold the appropriate temperature on the seventieth stage.

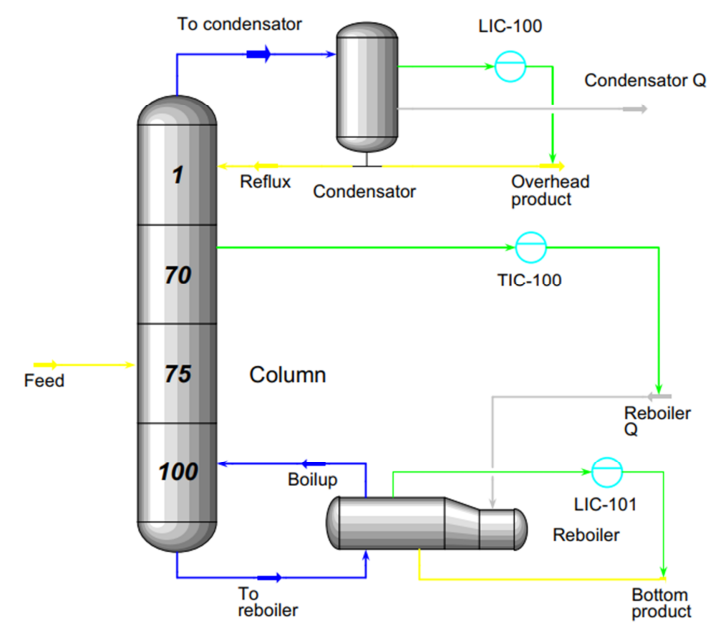

Fig. 3. First dynamic model of the system in Unisim Design software

In the first case, the mass flow of the feed stream was increased gradually. The results (Fig. 4) showed that the higher feed stream did not have influences on the 
temperature of the seventieth stage. However, the rectification column had geometry barriers. More than $63000 \mathrm{~kg} / \mathrm{h}$ feed stream caused flooding on the stages and it caused a malfunction in the system. In Fig. 4, Fig. 5, Fig. 8 and Fig. 9 the horizontal lines show the decreased or increased values of the mass flow of the feed progressing in time.

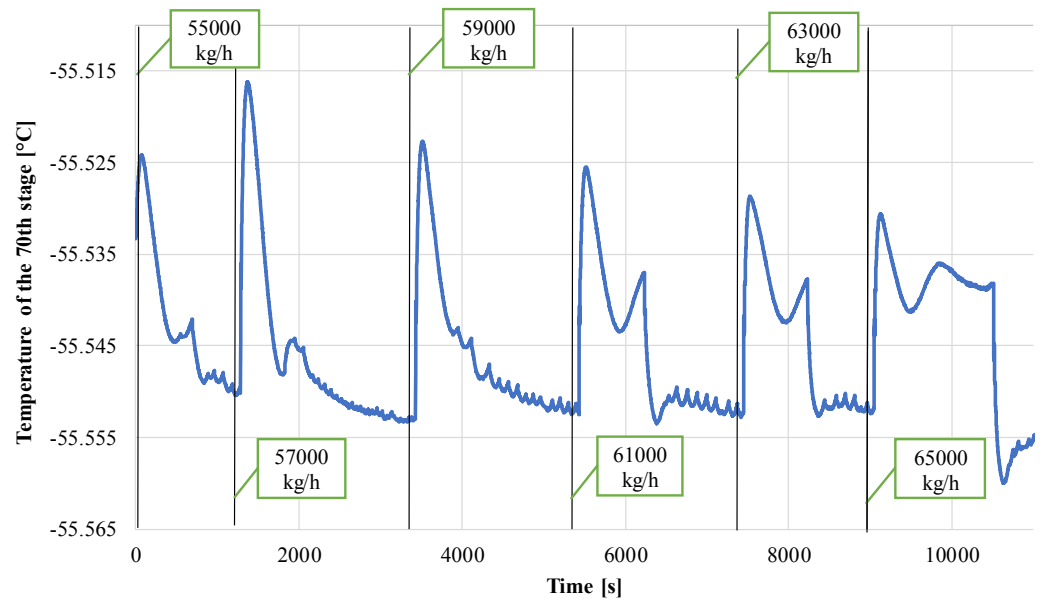

Fig. 4. The modification of the temperature, when the mass flow of the feed stream increased

Therefore, the mass flow of the feed stream was decreased gradually. The results of the simulations (Fig. 5) showed that less feed did not cause high-temperature modification in the system.

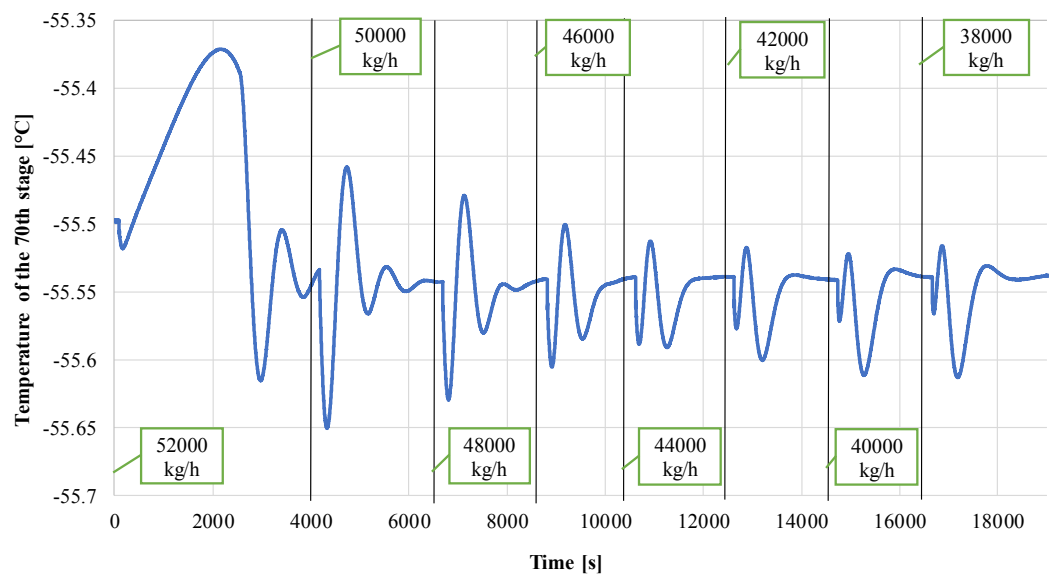

Fig 5. The modification of the temperature, when the mass flow of the feed stream decreased 
However, with this controlling structure, the less feed stream resulted in less ethylene purification in the overhead product (Fig. 6). In the chemical industry ethylene has high importance; therefore, in case of disturbances the distillate product of this rectification column should be high purified ethylene.

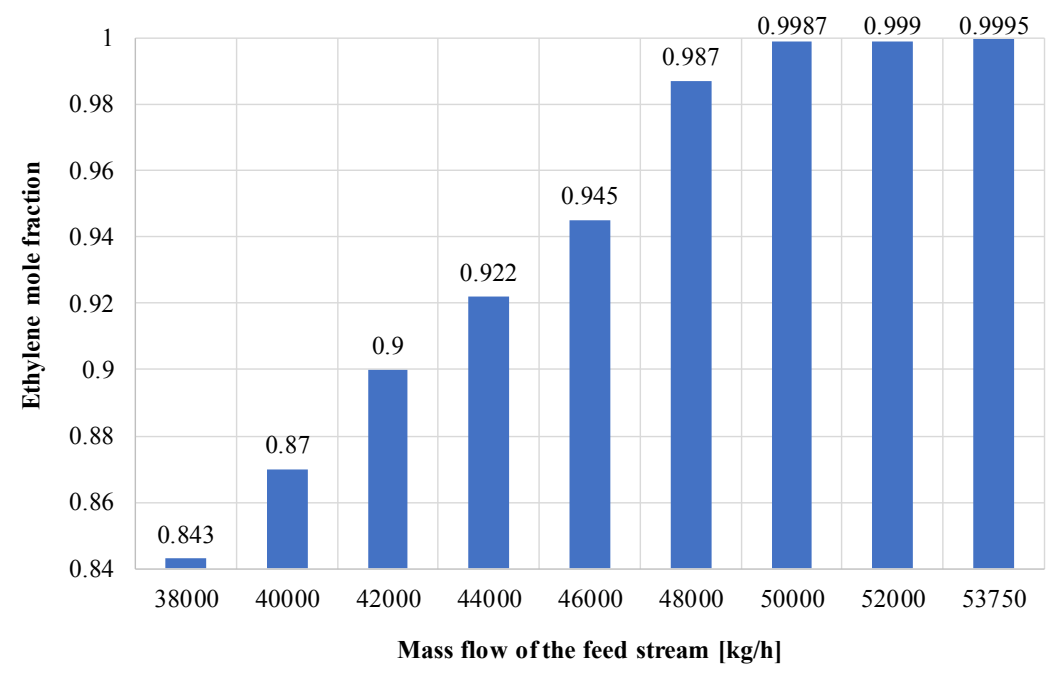

Fig. 6. Decreasing of the ethylene mole fraction in the distillate in case of the mass flow of the feed stream decreased

\subsection{Second investigated dynamic simulation model}

According to the previous statement, in this study another controlling structure was investigated with four PI controllers, the extra one was: a quality controller (XIC-100): this controlled the ethylene mole fraction in the distillate; the manipulated parameter was the mass fraction of the reflux stream (Fig. 7).

Two types of disturbances were also investigated in this case: increasing and decreasing gradually the mass flow of the feed stream. The behavior of the rectification column was investigated: the influences of the disturbances on the temperature of the seventieth stage and on the purification of the distillate.

Firstly, increasing the mass flow of the feed stream was investigated. The simulations showed (Fig. 8) that with this controlling structure, nor the temperature of the seventieth stage, neither the ethylene mole fraction of the overhead product modified. However, over the $65500 \mathrm{~kg} / \mathrm{h}$ value of the mass flow of the feed stream caused flooding on the stages and malfunction in the rectification column.

After that, the decreasing of the mass flow of the feed stream was also investigated in this controlling structure. In this case (Fig. 9) the highest temperature modification was investigated, but this changing was also not significant. Less than $46000 \mathrm{~kg} / \mathrm{h}$ mass flow of the feed stream caused a malfunction in the system: there were flooding on the stages of the stripping zone and the ethylene was lower than the required value. 


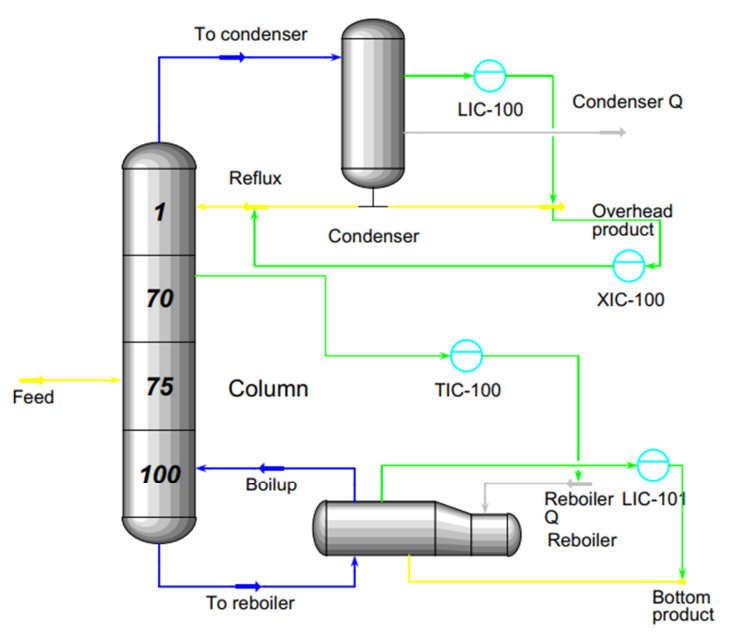

Fig. 7. Second dynamic model of the system in Unisim Design software

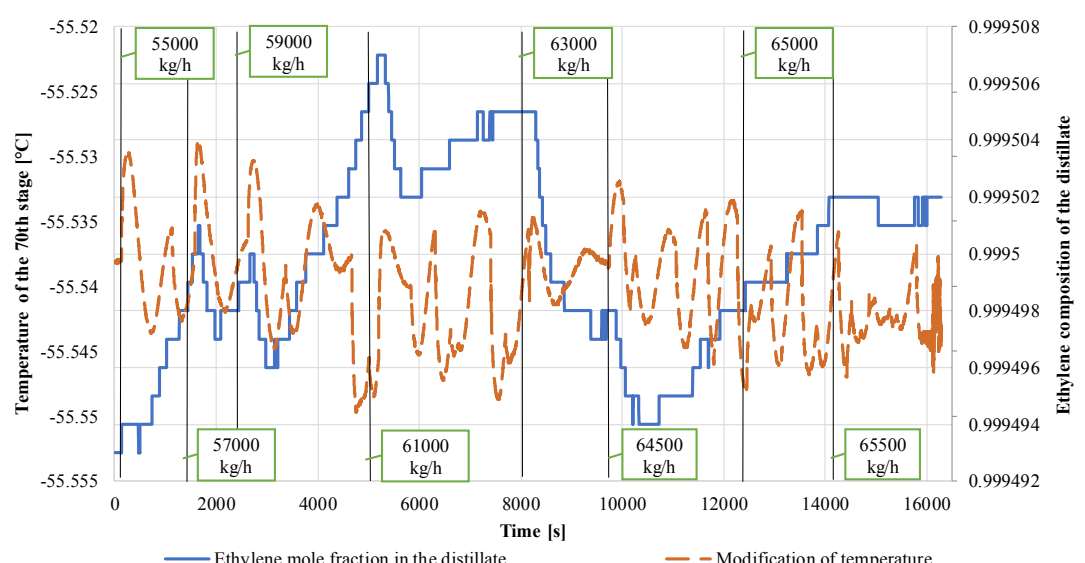

Fig. 8. The modification of the temperature and ethylene purification, when the mass flow of the feed stream increased

\section{Conclusion}

To summarize this study, it can be mentioned that the modification of the mass flow of the feed stream had influenced the behavior of the column. Lower feed stream caused lower, while higher feed stream caused higher temperatures. With only the temperature controlling the lower mass flow of the feed stream did not cause required product, while 
the higher mass flow of the feed stream caused flooding. Therefore, the separation did not operate, or its quality was not the desired one.

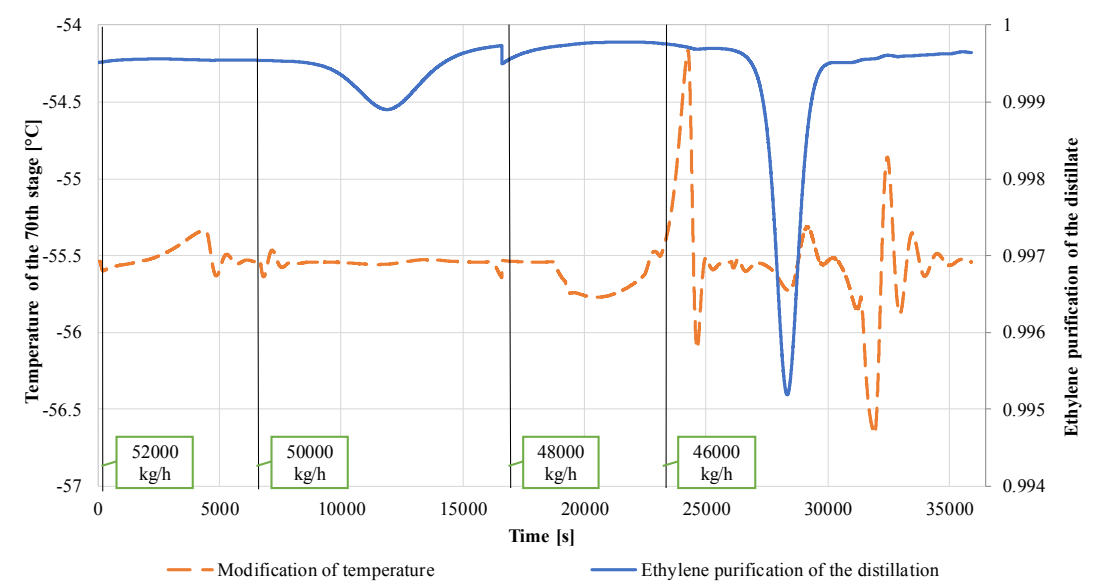

Fig. 9. The modification of the temperature and ethylene purification, when the mass flow of the feed stream decreased

With the temperature and composition controlling structure the lower and higher mass flow values of the feed stream had geometry barriers. However, with this lastmentioned structure, the desired quality of the overhead product can be reached.

The further goal is to investigate the behavior of the system in case of other disturbances, for example what will happen if the composition of the feed stream changes.

\section{Acknowledgements}

This research was supported by the ÚNKP-19-3 New National Excellence Program of the Ministry for Innovation and Technology.

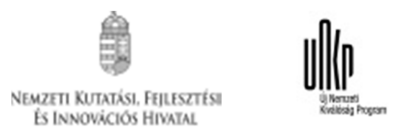

\section{Open Access statement}

This is an open-access article distributed under the terms of the Creative Commons Attribution 4.0 International License (https://creativecommons.org/licenses/by/4.0/), which permits unrestricted use, distribution, and reproduction in any medium, provided the original author and source are credited, a link to the CC License is provided, and changes - if any - are indicated. (SID_1) 


\section{References}

[1] Skogestad S. Dynamics and control of distillation columns, A tutorial introduction, Trans. Chemica Engineering Research and Design, Vol. 75, No. 6, 1997, pp. 539-562.

[2] Petrik M., Szepesi G., Jármai K. CFD analysis and heat transfer characteristics of finned tube heat exchangers, Pollack Periodica, Vol. 14, No. 3, 2019, pp. 165-176.

[3] Firmino C. K., Da Silva C. A. C., Da Silva M. M., Biaggi P. N., Ferreira N. L. Dynamic simulation of an industrial distillation column, The Journal of Engineering and Exact Sciences, Vol, 5, No. 1, 2019, pp. 143-147.

[4] Kniese D., Haber R., Volk U., Hahn R. Optimized multivariable predictive control of an industrial distillation column considering hard and soft constraints, IFAC Procedings Volumes, Vol. 36, No. 8, 2003, pp. 239-244.

[5] Truong H. S., Ismail I., Razali R. Fundamental modeling and simulation of a binary continuous distillation column, International Conference on Intelligent and Advanced Systems, Manila, Philippines, 15-17 June 2010, pages 1-5.

[6] Baiesu A. Controlling a complex propylene-propane distillation column using a robust method suitable for simple processes, Rev. Chim, Vol. 64, No. 4, 2013, pp. 445-450.

[7] Marcsa D., Kuczmann M. Closed loop control of finite element model in magnetic system, Pollack Periodica, Vol. 10, No. 3, 2015, pp. 19-30.

[8] Taqvi S. A., Tufa D. L., Muhadizir S. Optimization and dynamics of distillation column using Aspen Plus ${ }^{\circledR}$, Procedia Engineering, Vol. 148, 2016, pp. 978-984.

[9] Haber R., Bars R., Schmitz U. Predictive control in process engineering from the basis to the applications, Wiley, 2011.

[10] Gerzson M., Pletl S. Control Engineering, (in Hungarian) Typotex, Budapest, 2011.

[11] Skogestad S., Jacobsen E. W., Lundstrom P. Selecting the best distillation control structure, IFAC Proceedings Volumes, Vol. 22, No. 8, 1989, pp. 315-322.

[12] Mizsey P. Process control systems, (in Hungarian) Budapest, Typotex Kiadó, 2011.

[13] Shi M., Lin C. C. H., Kuznicki T. M., Hashisho Z., Kuznicki S. M. Separation of a binary mixture of ethylene and ethane by adsorption on Na-ETS-10, Chemical Engineering Science, Vol. 65, No. 11, 2010, pp. 3494-3498.

[14] UniSim ${ }^{\circledR}$ Design, User Guide, User Guide, 2009.

[15] Jaubert J. N., Privat R. Relationship between the binary interaction parameters (kij) of the Peng-Robinson and those of the Soave-Redlich-Kwong equations of state: Application to the definition of the PR2SRK model, Fluid Phase Equilibrium, Vol. 295, No. 1, 2010, pp. 26-37.

[16] Nasri Z., Binous H. Rigorous distillation dynamics simulations using a computer algebra, Computer Applications and Engineering Education, Vol. 20, No. 2, 2012, pp. 1-10.

[17] Cho Y., Kim B., Kim D., Han M., Lee M. Operation of divided wall column with vapor sidedraw using profile position control, Journal of Process Control, Vol. 19, No. 6, 2009, pp. 932-941. 